\section{Cultural competency and culturally safe clinical care}

We enjoyed reading the timely and valuable commentary by Katz and colleagues. ${ }^{1}$ However, a major limitation of this commentary is that the authors fail to address the issue of substance abuse that affects the First Nations, despite its high prevalence and the enormity of its consequences to these communities. Instead, they focused their commentary exclusively on suicides. It is important to note that First Nations communities with higher than average percentages of drug addiction and chemical dependency have higher incidences of suicide and violent crimes. ${ }^{2,3}$

The First Nations Regional Health Survey (2008-2010) showed that among First Nations people aged 18 and older living on reserve or in northern First Nations communities, $4.7 \%$ reported past-year use of illegal (heroin) or prescription opioids (including morphine, methadone and codeine) without a prescription. ${ }^{4}$

Meanwhile, recent First Nations Health Authority data showed that First Nations people in British Columbia are five times more likely to have an overdose and three times more likely than the general population to die from an overdose. ${ }^{5}$ Fourteen percent of those who overdose (with some overdoses resulting in death) are First Nations, even though First Nations people represent only $3.4 \%$ of the $\mathrm{BC}$ population. The statistics confirm that First Nations communities have been disproportionately affected by the current opioid crisis. ${ }^{5}$

Further, the authors of this timely commentary ${ }^{1}$ emphasize the importance of increasing the number of First Nations physicians in Canada. We wholeheartedly appreciate this proposal; it is also a part of concerted efforts by the Canadian government, the Association of Faculties of Medicine of Canada and the Indigenous Physicians Association of Canada to increase the number of physicians in Canada through a variety of initiatives.

Even though the commentary ${ }^{1}$ highly values First Nations health care workers as a potential solution for contemporary complex health care problems in the First Nations communities, it is doubtful that this strategy alone would solve the entire problem effectively and efficiently. Available research also suggests that it is difficult to retain nursing graduates within the First Nations communities to serve their specific communities. These graduates often consider their degree as a "way out" of the isolated reserves and into a setting where more competitive salaries are offered in exchange for their skills. ${ }^{6}$

There are other obstacles, and related research suggests that it is not easy to recruit First Nations students to medical schools. ${ }^{7}$ Until at least this problem of recruiting is resolved, it is important that current medical training includes and gives due importance to training culturally competent doctors. This would no doubt reap enormous benefits, as more than half of Canada's 1.3 million First Nations people live in urban areas.

Cultural competency - and effective implementation of that cultural competency - is a national challenge with consequences for both individual patients and Canadian society, requiring appropriate knowledge about different cultures, cultural norms and cultural values.

There is an urgent need to explore specific evidence about which strategies are most effective for improving both culturally competent health care and delivery of culturally safe clinical care to First Nations communities.

Developing core competencies for undergraduate, postgraduate and continuing medical education specifically to enhance cultural competency and meaningfully advance culturally safe clinical care in the delivery of health services to First Nations communities is necessary. This, in fact, appropriately reflects Canada's accreditation standards for medical schools, which stress that doctors would be best prepared to deliver health care to a diverse population if they are educated in a diverse environment, where they are exposed to other cultures.

However, we need to understand that clinicians become culturally competent only with the support and encouragement of the health care system in which they operate. Therefore, it is equally important that we are serious in our thinking on how to build health care institutions that are sustainably culturally competent throughout Canada. ${ }^{8}$

\section{Ediriweera Desapriya PhD}

Research Associate, Department of Emergency Medicine, University of British Columbia, Vancouver, BC

\section{Vahid Mehrnoush MD}

Research Coordinator, Vancouver General Hospital, Vancouver, BC

\section{Aki Nilanga Bandara}

Research Assistant, Vancouver General Hospital, Vancouver, BC, Burnaby, BC

- Cite as: CMAJ 2018 January 22;190:E84. doi: $10.1503 / \mathrm{cmaj} .733435$

\section{References}

1. Katz A, Enns J, Kinew KA. Canada needs a holistic First Nations health strategy. CMAJ 2017;189: E1006-7.

2. Resolution no. 57/2011: Support for Akwesasne leadership in addressing state of crisis regarding substance abuse. Ottawa: Assembly of First Nations; 2011.

3. Sullivan L; National Native Addictions Partnership Foundation. Synopsis of First Nations substance abuse issues: developed for use by the RNAO. Toronto: Registered Nurses Association of Ontario; 2015.

4. First Nations Regional Health Survey (RHS) 2008/10: National report on adults, youth and children living in First Nations communities. Ottawa: First Nations Information Governance Centre (FNIGC); 2012.

5. Wadhwani A. First Nations people in B.C. three times more likely to die of overdose: New figures paint a disturbing image of the overdose crisis for First Nations people. Salmon Arm Observer 2017 Aug. 3.

6. Goraya J. Access to health care on Aboriginal reserves. Toronto: The Public Policy \& Governance Review; 2016. Available: https://ppgreview.ca /2016/04/06/access-to-health-care-on-aboriginal -reserves-2/ (accessed 2017 Sept. 8).

7. Petch J, Tepper J, Konkin J. Canadian medical schools struggle to recruit Aboriginal students. Toronto: Healthy Debate (La Ki Shing Knowledge Institute, St. Michael's Hospital); 2013. Available: http://healthydebate.ca/2013/05/topic/ quality/recruitment-of-aboriginal-health-care -workers (accessed 2017 Sept. 8).

8. Brach C, Fraser I. Reducing disparities through culturally competent health care: an analysis of the business case. Qual Manag Health Care 2002;10:15-28.

Competing interests: None declared. 